\title{
Die Axillaris der Papilionidae. Eine Entgegnung an Herrn F. Bryk.
}

Von H. Stichel, Berlin-Lichterfelde.

Es gibt Kritiken, deren Inhalt die Ignoranz der Person ihres Autors reflektiert. Man kann dies in der Regel schon an der Mafslosigkeit der Sprache erkennen, so bei F. B ryk in seinem Artikel „Über das Abändern der Rippenkonfiguration im Genus Parnassius" Archiv f. Naturgesch. v. 82, 1916, Abt. A, p. 42. Der Autor nennt ihn „Beiträge zur Entwicklung des Rhopalocerengeäders"; es deucht mich, dals hiervon in den an gedrehten Worten besonders reichen Darstellungen anormaler Geädertypen nicht viel zu finden ist, was dem Sinn dieser Titelerweiterung gerecht werden kann, es soll aber nicht meine Aufgabe sein, dies zu erörtern, sondern ich beschränke mich auf die Abweisung einer von seltener Selbstüberhebung getragenen Polemik Herrn Bryks.

Gegenstand ist die Rippe (besser Ader) $\mathrm{Ax}_{2}$ im Vordertügel der Schmetterlinge. Herr B. schreibt, 1. c. Fufsnote p. 42, dafs sie das am meisten auffallende Merkmal aller Papilioniformia (recte Papilioniformes) sei, nur beim Herrn Stichel gäbe es Papilioniden, die keine $\mathrm{Ax}_{2}$ besitzen, der "Thaitites" hätte er in Genera Insectorum fase. 59 keine $A x_{2}$, hineingezeichnet ", auch bei Charakterisierung der Gattung Zerynthia sei sie mit keinem Worte erwähnt (l. c. p. 7).

Beim Schlufs dieser Kritik anfangend, so ergibt eine Nachprüfung, dafs die Behauptung falsch ist. Im angezogenen Text steht wörtlich „, an der Wurzel (nb: der Submediana) ein kurzer, in den Hinterrand mündender Nebenzweig". Dafs sich diese Bezeichnung mit $A x_{2}$ deckt, mufste Herr $B$. wissen, denn er bemängelt sie später, worauf ich noch zurückkomme. Dieser Punkt wäre aber auch sonst bedeutungslos, weil die Ader in dem l. c. t. 1 fig. $1^{b}$ gebrachten Geäderschema deutlich ausgedrückt ist.

Die voraufgehende Behauptung Herrn Bryks ist auch nur bedingungsweise richtig. Ich habe dem Geäderschema der fossilen Gattung Thaites Scudder (Heer i. 1.) (falso Thaitites nach Bryk) die Ader nicht hineingezeichnet, weil das Bild eine vergröfserte Kopie nach S. H. Scudder ist. Auch dies mufste Herr B. erkennen, wenn er den Stoff beherrschte, zum Utberflufs ist auch ans der Tafelerklärung l. c. p. 26 die Tatsache zu ersehen. Für den Mangel mürste also $\mathbf{S} \mathbf{c u d d e r}$ verantwortlich gemacht werden. Damit soll aber nicht anerkannt werden, dafs Herrn Bryks Rüge an sich gerechtfertigt ist, denn im Petrefakt ist die hintere 
Flügelpartie zerstört, und der Autor konnte dem Bilde keine Ader zufügen, die nicht zu erkennen oder durch eine sichere Kombination zu rekonstruieren ist. Es mufs Herrn Bryk überlassen werden nachzuweisen, dals das Original der Versteinerung aus dem Tertiär eine $\mathrm{Ax}_{2}$ gehabt hat!

Nun bezeichnet Herr B. die Ader als ein „Adelswappen“ der Papilionidae, er hat dabei „übersehen", dals sie auch bei einer grofsen Reihe anderer rezenter Gattungen, wenn auch in modifizierter Anlage, auftritt. Sie mündet als hinterste Ader des Flügels in die Submediana $\left(\mathrm{Ax}_{1}\right)$ und wird dann als "Spaltung" oder "rücklaufender Ast" bezeichnet, so bei Ithomiidae, vielen Pieridae, Euploeidae, etlichen Nymphalidae (Clothilda), Satyridae (Haetera, Pierella), Lycaenidae (Thestor, Thecla, Hypolycaena), Riodinidae, auch unter den Heterocera bei Cossus, Zygaena, Demas, Sesia, Ourapteryx, Boarmia u. a. In einigen Fällen liegt sie aber auch frei in der Membran, ohne allerdings den Hinterrand zu erreichen, so bei Spilosoma. Es mag dem Urteil des Lesers überlassen bleiben, ob der Ader hiernach der Wert für die Papilionidae beizumessen ist, der in dem Worte „Adelswappen" ausgedrückt ist, zumal ihre Ausbildung in dieser Familie selbst nicht konstant ist, denn es gibt Fälle, in denen sie den Hinterrand nicht ganz erreicht (Luehdorfia) und in denen sie mit der Submediana $\left(\mathrm{Ax}_{1}\right)$ im Anfang verwachsen ist, also aus dieser abzweigt, so z. B. bei dem afrikanischen Papilio tyndaraeus F. Wenn Herrn Bryk diese Verbältnisse auch bei den exotischen Genera mangels ausreichender Übersicht unbekannt geblieben sind, so konnte man verlangen, dafs er wenigstens die paläarktischen Vertreter der Ordnung in den Horizont seiner Kenntnisse einschlofs. Nachrichtlich sei übrigens noch erwähnt, dals die Ader auch in vollkommener, d. i. frei in den Hinterrand auslaufender Form im Hinterflügel wohl der meisten rezenten Lepidopteren-Gattungen auftritt, sie wird dort als "Hinterrandader" bezeichnet, und in einem mir bekannten Falle erscheint sogar noch eine $\mathbf{A x}_{3}$ als „Endader" bei der Gattung Morphopsis Oberth. (Gen. Ins. v. 39 p. 5 t. 1 f. 4 ).

Wir kommen nun zu der Erklärung der ominösen $A x_{2}$, weil Herr Bryk bezüglich meiner weiter erklärt, dals „dieser entomologische Dilettant" überhaupt keine wissenschaftliche Bezeichnung kennt, sie heilst bei ihm bald Nebenast, Nebenader oder Nebenzweig. Was das Persönliche angeht, so summiere ich es weiter unten, hier zunächst nur das Sachliche. Voraussetzung für das Urteil ist, dals eine deutsche oder im weiteren eine in der Landessprache des Verfassers gebrauchte Bezeichnung keine wissenschaftliche ist. Wie verfehlt solche Ansicht ist, erhellt u. a. aus der Benutzung von Ausdrücken wie Vorderrand für Costa, Hinter- 
oder Innenrand für Dorsale, Hinterrandader für $\mathbf{A x}_{\mathbf{2}}$ (im Hinterflügel, s. oben) oder gar eine blofse Numerierung der Adern (oder Rippen) und schlielslich noch das Wort Rippe selbst statt Trachee, das Herr Bryk in der von Enderlein entlehnten hybriden $\mathrm{Zu}-$ sammensetzung „Rippenkonfiguration" gebraucht. Es bleibt also sinngemäls nur der Einwand haltbar, dals ich für diese als $\mathbf{A x}_{2}$ bezeichnete Ader keine altsprachliche, sondern eine Bezeichnung in meiner Muttersprache angewendet oder beibehalten habe. Diesem Brauch folgen aber nicht nur deutsche, sondern auch namhafte ausländische Lepidopterologen, so charakterisiert F. Moore in Lepidopt. indica v. 5 p. 102 die Papilionidae u. a. wie folgt: „submedian with a lower basal short branch or spur extending towards the posterior margin ". In diesem "short branch" erkennen wir Herrn Bryks $\mathrm{Ax}_{2}$.

Was versteht man also unter $\mathrm{Ax}_{2}$ ? Aus dem Text des Brykschen Artikels p. 42 ersehen wir, dals es eine Abkürzung für Axillaris ist, ein Name, den Herr Bryk auch von Enderlein (Zool. Jahrb. Anat. Ont. v. 16 p. 15, 1912) entlehnt hat. Enderlein hat ihn eingeführt, um die morphologisch verschiedenartigen, als Analadern zusammengefalsten Adern „besser zu charakterisieren". Die Anales Comstocks, zu der jene "Nebenader" der Papilionidae als Analis 3 gezählt wurde, zerfallen deswegen in Äste der Analis und der Axillaris. Dals diese Aufteilung besonders glücklich ist, will mir nicht scheinen, jedenfalls verbleibt dann die Bezeichnung Analis nur der ersten (vordersten) der drei ursprünglich darunter verstandenen Adern, Analis 2 und 3 werden zu Axillaris 1 und 2. Jene, die Analis 1, hat dann für die Rhopalocera nur, noch einen ontogenetischen Wert oder präimaginalen Sinn, weil sie im Tracheensystem der fertigen Imago völlig fehlt oder nur als kurzer "Sporn" nahe der Wurzel der Mediana des Vorderflügels erhalten ist, so bei den Heliconiidae, Dionidae, Morphidae u. a. Ihre Lage ist im übrigen nur durch eine mehr oder weniger deutliche Falte in der Membran angedeutet. Abgesehen hiervon ist die Einführung des Namens Axillaris deswegen nicht einwandfrei, weil für $\mathrm{Ax}_{1}$ bereits lange vorher der altsprachliche Name Submediana oder auch Dorsalader in Gebrauch war. Der Ursprung des Wortes Submediana datiert wohl aus 1842 von Lefebure, der dafür in seiner Muttersprache "Sousmediane" sagt, und dies ist dann latinisiert worden. So hat $u$. a. auch schon Felder 1861 (Nova acta v. 28) dafür die Abkürzung SM gewählt und Jordan (Nov. Zool. v. 13 p. 745) sich zu Submediana entschieden, indem er die sogenannte Faltenader, also die Spuren der Analis, nach Comstock, als $\mathbf{S M}_{1}$, die Analis 2 nach Comstock (= Submediana Felder, Schatz u. a., Axillaris 1 
nach Enderlein) als $\mathrm{SM}_{2}$, endlich die Analis 3 nach Comstock (= Axillaris 2 Enderlein) als $\mathrm{SM}_{3}$ bezeichnet.

Von diesen Tatsachen geleitet und in Hinblick darauf, dafs es bei der Analysierung des Flügelgeäders in der Hauptsache auf das Bild ankommt, das wir im Imaginalstadium sehen, habe ich mich, wie ich dies auch in Genera Ins. v. $12^{b}$ p. 395 besonders hervorhob, der empirischen Methode Herrich-Schäffers and Schatz' (Röber) angeschlossen und dabei für Axillaris 2 (Enderlein) die hergebrachte deutsche Bezeichnung Nebenast (oder Nebenzweig bzw. Nebenader) der Submediana gewählt und für Axillaris 1 den prioritätsberechtigten Namen Submediana beibehalten. 0 . a. 0 . Seite 451 habe ich schliefslich noch eine vergleichende Übersicht der gebräuchlichen Geädernomenklatur, zu der die Enderleinsche allerdings nicht gerechnet worden ist, gegeben.

So kann also von einer Ignorierung von $\mathbf{A x}_{2}$ durch mich, wie Herr Bryk sich auslälst, gar nicht die Rede sein!. Wer bei diesen Erörterungen nun seine „Unkenntnis vor der ganzen deutschen Wissenschaft dokumentiert" (vgl. Bryk l. c. p. 43) und auf wen der weiterhin mir von Brykerteilte Ratschlag, sich besser zu bilden, anzuwenden ist, das will ich gern dem Urteil des Lesers überlassen. Nachdrückliche Verwahrung aber will ich einlegen gegen den Appell des Polen Bryk an die deutsche Wissenschaft! Im blinden Zorn über früher von mir erfahrene Abweisungen hält es Herr B. auch noch für angebracht, meinen Beruf in wegwerfender und irreführender Apostrophierung in die Debatte zu ziehen. Geschmacklosigkeiten dieser Art sind deutschem Wesen fremd. Man wird sie ihm als Ausländer zugute rechnen müssen, es muls aber wunder nehmen, dafs der Herr Redakteur des "Archivs" es nicht für nötig befunden hat, derartige gegen die guten Sitten verstofsende Ausfälle zu verhindern, denn diesen schützt seine Nationalität nicht, weil er eingedenk sein mufs dessen, dafs er deutsche Gastfreundschaft genielst und deutsches Brot ifst!

Anders verhält es sich mit dem vorher zitierten, auf mich angewendeten Epitheton „entomologischer Dilettant". Hiergegen ist grundsätzlich nichts einzuwenden, wenn Herr Bryk ihm auch einen anderen, geringschätzenden Sinn beilegen möchte, wie in dem Wort ausgedrückt ist. Welchen Anteil gerade der Dilettantismus an den Erfolgen im besonderen der entomologischen Wissenschaft gehabt hat, lehrt uns deren Geschichte! Ob und inwieweit meine dreifsigjährige dilettantische Tätigkeit an diesen Erfolgen bescheidenen Anteil gehabt hat, soll dem Urteil Berufener überlassen bleiben. $\mathrm{Zu}$ diesen zählt Herr Bryk nicht, es wäre aber interessant zu erfahren, zu welcher Kategorie von Entomo- 
logen sich Herr Bryk, der m. W. von Beruf Maler ist, sich rechnet!

Auch in dem Schlufssatz der zitierten Fufsnote greift Herr Bryk fehl. Es ist mir gar nicht eingefallen, ihm vorzuschreiben, wie er seine Artikel verfassen soll, ebensowenig habe ich mir angemarst, im Namen der deutschen Wissenschaft zu sprechen, ich habe in dem betreffenden Referat (Zeitschr. f. wiss. Insektenbiol. v. 11 p. 105) nur betont, dars Artikel mit so banalen Überschriften und mit ebensolchem Inhalt, wie sie Herr Bryk publiziert hat, nicht in Blätter gehören, die Anspruch auf wissenschaftliche Beachtung erheben und dals die von ihm gepflegten Stilblüten geradezu ein Hohn auf den Ernst und die Würde deutschen Forschungsgeistes seien, wie auch zu bezweifeln sei, dafs des Schreibers Reife diesen Eigenschaften gewachsen ist. Ich brauche diese Worte nur zu unterstreichen, um sie als Endsumme meiner Abweisung zu buchen, womit ich gleichzeitig die Kontroverse mit Herrn Bryk endgültig beschliefse.

\section{Kommt Carabus auratus L. in Ostpreufsen vor?}

Von F. Schumacher, Charlottenburg.

Als ich mich mit der östlichen Verbreitungsgrenze des Carabus auratus beschäftigte, machte ich darauf aufmerksam, dals dieser Käfer in Ostpreufsen augenscheinlich ein kleines abgeschlossenes Verbreitungsgebiet besitzt. Die Richtigkeit der Angabe ist neuerdings bestritten worden, so namentlich von $H$. v. L e ngerken. Er hatte bei den Königsberger Entomologen Erkundigungen eingezogen, die negativen Erfolg hatten. Im folgenden will ich die Gründe und Quellen anführen, die mich veranlassen, den Käfer als ostpreufsisch anzusprechen. Ku gella n führt ihn von Osterode als "selten auf dem Acker" an. Illiger hat ihn von dort in Händen gehabt, wie in der Bearbeitung des Verzeichnisses (1798) vermerkt ist. Siebold (1847) und Lentz (1857, 1879) wiederholen die Angabe. Fritzen (1865) bezeichnet ihn als sehr selten in "Preufsen", indem er sich auch wohl nur auf Kugellans Angabe stützt. Ich gebe gern zu, dafs diese alte von K. herrührende Notiz unzuverlässig sein mag, aber seine Angabe wird durch eine andere sicher ernst zu nehmende gestützt, die ein Vorkommen in nächster Nachbarschaft bei Allenstein bekanntgibt. In einem an $\mathrm{Schils} \mathrm{ky}$ gerichteten Brief bemerkt $\mathrm{Cz}$ wa lina, dafs ersterer im Katalog das ? bei „Preufsen" streichen müfste: "Seidlitz hat ihn vor kurzem aus Allenstein za- 